\title{
ERRATUM - BJID 15(5)
}

In the article "Risk factors for mortality in Acinetobacter bateremia", Braz J Infect Dis. 2011;15(5):501-2, consider as corresponding author Hee Jung Yoon (yhj82222@gmail.com). 